\title{
Surface plasmon resonance enhancement of photoluminescence intensity and bioimaging application of gold nanorod@CdSe/ZnS quantum dots
}

\author{
Siyi Hu${ }^{1}$, Yu Ren², Yue Wang ${ }^{2}$, Jinhua $\mathrm{Li}^{2}$, Junle $\mathrm{Qu}^{3}$, Liwei Liư ${ }^{*}$, Hanbin Ma*1 \\ and Yuguo Tang ${ }^{1}$
}

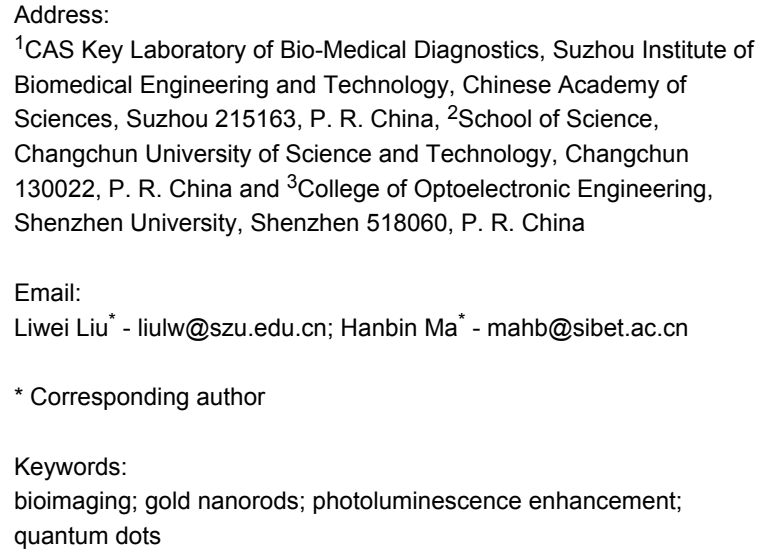

\begin{abstract}
Biological applications of core/shell near-infrared quantum dots (QDs) have attracted broad interest due to their unique optical and chemical properties. Additionally, the use of multifunctional nanomaterials with near-infrared QDs and plasmonic functional nanoparticles are promising for applications in electronics, bioimaging, energy, and environmental-related studies. In this work, we experimentally demonstrate how to construct a multifunctional nanoparticle comprised of CdSe/ZnS QDs and gold nanorods (GNRs) where the GNRs were applied to enhance the photoluminescence (PL) of the CdSe/ZnS QDs. In particular, we have obtained the scattering PL spectrum of a single CdSe/ZnS QD and GNR@CdSe/ZnS nanoparticle and comparison results show that the CdSe/ ZnS QDs have an apparent PL enhancement of four-times after binding with GNRs. In addition, in vitro experimental results show that the biostability of the GNR@CdSe/ZnS nanoparticles can be improved by using folic acid. A bioimaging study has also been performed where GNR@CdSe/ZnS nanoparticles were used as an optical process for MCF-7 breast cancer cells.
\end{abstract}

\section{Introduction}

In the past decades, quantum dots (QDs) have proven to be increasingly useful for their unique features [1-5]. The light emission from QDs can be easily tuned from the visible to the near-infrared (NIR) spectrum. QDs have emerged as the next generation of luminescence materials, and they have been widely used as nanoprobes for bioimaging and biosensing. As semiconductor nanocrystals, their large surface-to-volume ratio is advantageous and they show excellent quantum confinement 
for charge carrier and lattice mismatch. In such cases, the energy range of the QDs depends on the relative conduction and valence band offsets for the two materials, and this characteristic can enable many chemical materials or biomolecules to conjugate with them [6-12]. Research conducted to date shows that the fluorescence intensity of QDs changes when other chemical materials or ions are added. The CdSe/ZnS heterostructures of QDs are of interest due to their high quantum efficiency [13-15].

Furthermore, the heterostructure formed with metals and semiconductors, i.e., plasmonic, composite QD nanostructures, provides another efficient way to tune the unique optical properties. In the past decades, much attention has been given to the development of metal-enhanced optical properties. Some researchers have noted that certain metal materials play a role in the enhancement of fluorescence in QDs, especially gold nanorods (GNRs) and $\mathrm{Cu}$ or $\mathrm{Ca}^{+}$ion binding of QDs [16-19].

GNRs possess two plasmonic resonance bands - a longitudinal band and a transverse band. These bands correspond to the elec- tron oscillations along the long and short axes of the particle. Their surfaces can be easily modified with other chemicals due to the large area-to-volume ratio of GNRs. Also, there is a positive charge coating on the GNR surface, so it is easily conjugated with QDs due to the negative charge of the QD surface. The excellent stability and biocompatibility of GNRs has been reported by several researchers, and they are being investigated as a probe for photothermal therapy in nanomedicine. The presence of longitudinal surface plasmon resonance (LSPR) provides GNRs with richer optical properties, which lead to local field, Raman, and fluorescence enhancement. When QDs bind with GNRs, the fluorescence intensity of the QDs is enhanced by the near-fired plasmonic resonance from the GNR surface and their photoluminescence (PL) emission increases [20-23].

Richard A. Vaia and co-authors reported a five times higher fluorescence enhancement by organizing QDs on GNRs, and Anjali Kshirsagar et al. reported the electronic structure of freestanding and gold-attached passivated CdSe nanorods [24,25]. These studies covered the synthesis of GNR@CdSe/ZnS nano-

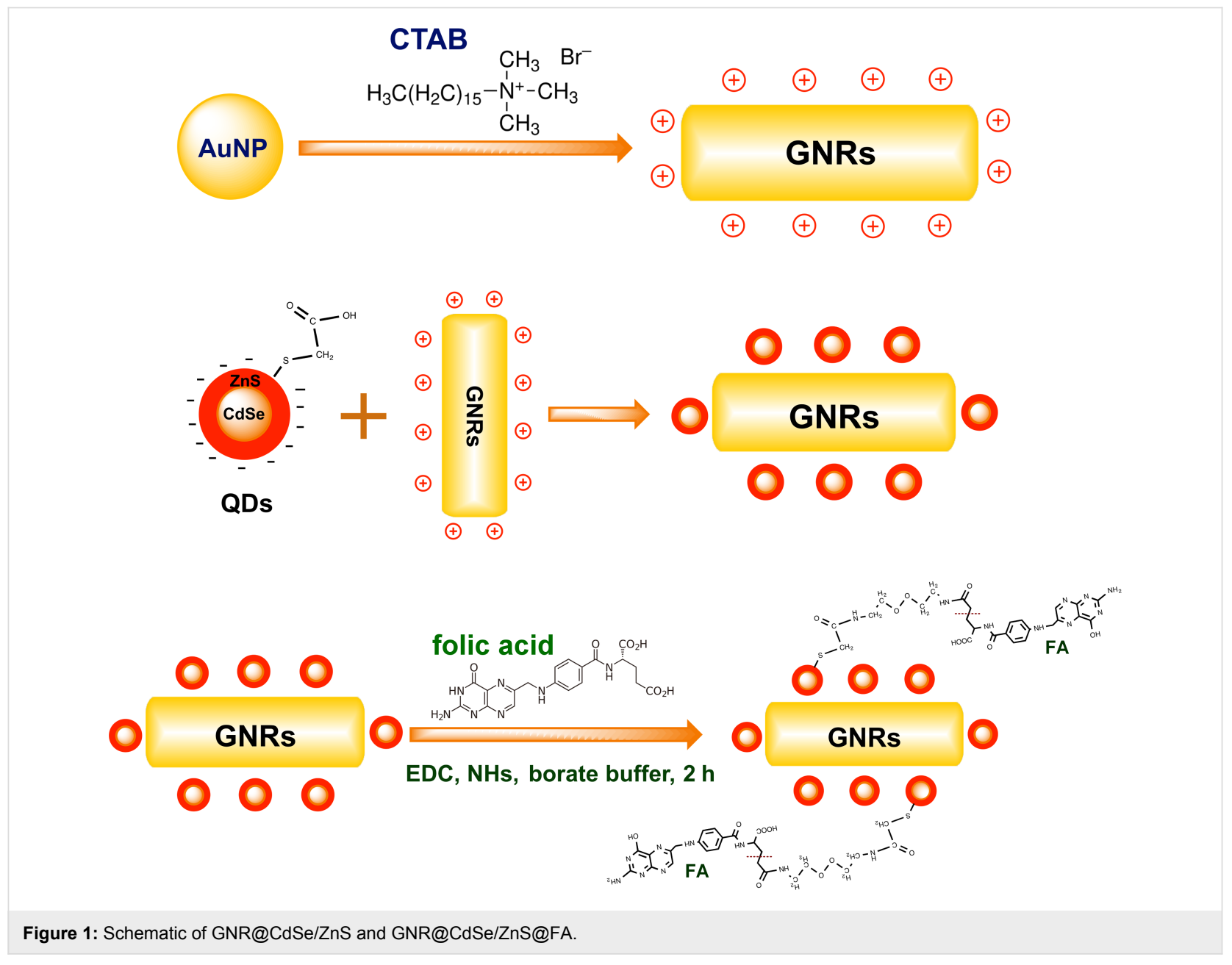


particles using different methods; however, most of these synthesis methods are complicated, and it is difficult to reproduce their preparation and desired applications. It is desirable to find a simple method to synthesize GNR@CdSe/ZnS nanoparticles in aqueous-phase which exhibit high PL emission and optimize the bioconjugated surfaces of these nanoparticles for biological application and therapy of human diseases [26,27].

In this work, we demonstrated a novel GNR@CdSe/ZnS multimodal nanostructure in aqueous phase. We chose $\mathrm{CdSe} / \mathrm{ZnS}$ QDs as a PL contributor due to its high degree of brightness, excellent photostability, and good spectral overlap with GNRs. We then used the GNRs to enhance the PL intensity of the CdSe/ZnS QDs. The PL from GNR@CdSe/ZnS nanoparticles is approximately four times more than that from $\mathrm{CdSe} / \mathrm{ZnS}$ QDs. Finite difference time domain (FDTD) simulations were also conducted to understand the plasmon coupling effect on PL enhancement. Additionally, we investigated the PL signal from a single particle, which indicated a stronger PL intensity compared to that of $\mathrm{CdSe} / \mathrm{ZnS}$ QDs alone. We also prepared the GNR@CdSe/ZnS modified with folic acid (FA) in cell culture for biological applications. These studies indicated that these multifunctional nanoparticles were of low toxicity and had bright luminescence, which make them suitable for biosensor and optical detection studies.

\section{Results and Discussion}

The scheme for the synthesis of GNR@CdSe/ZnS and GNR@CdSe/ZnS@FA is illustrated in Figure 1. Firstly, the $\mathrm{CdSe} / \mathrm{ZnS}$ QDs bind with GNRs kept at a $5 \mathrm{~nm}$ distance using the combined strong electrostatic adsorption. Secondly, FA was conjugated with this composite nanoparticle for biological applications, where the FA renders the nanoparticle useful for the specific targeting of cancer cells [28,29].

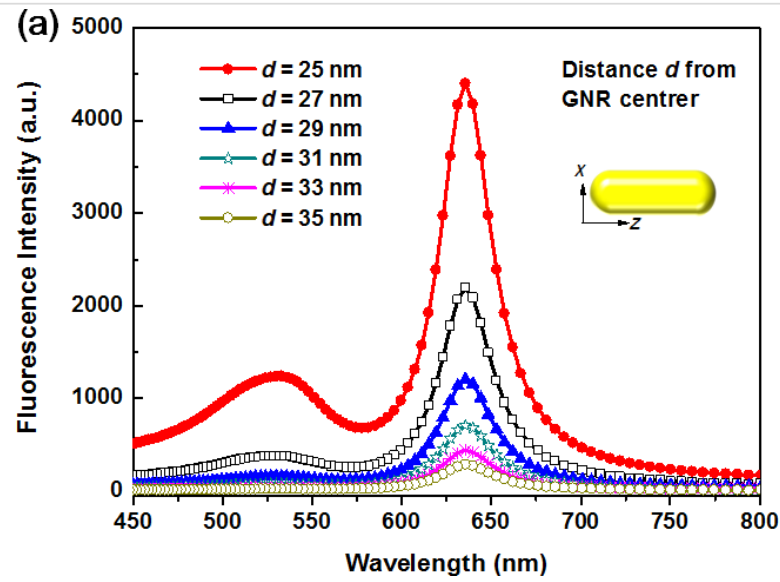

According to current knowledge, when bulk semiconductor nanomaterials are irradiated with light of a certain wavelength, the valence electrons transition back to the conduction band. Most of the conduction band electrons fall back into the deep electron traps and nonradiative transitions take the form of quenching. Semiconductor QDs with relatively shallow surface electron traps and holes can easily capture electrons to the conduction band. At the same time, the transition radiation returns to the valence band, emitting a photon. The PL characteristics are, therefore, better than in materials which have deep traps in the bulk semiconductor material. This finding suggests that when metal or chemical materials are doped with QDs, the number of surface trap states (which can lead to exponential PL decay) is affected by the surface passivation and by the degree of quantum confinement. In CdSe/ZnS QDs with higher defect densities, binding with GNRs having very heterogeneous energetic metal-derived states showed that their PL could be characterized by band edge dispersion in different sizes of $\mathrm{CdSe} / \mathrm{ZnS}$ QDs and GNRs and that the PL contribution varied in these new states. The physical or chemical origin of these features was not clear, and this will be a subject of future research. We chose the longer absorption peak of the GNRs as equivalent to the QD PL emission peak. When the QDs and GNRs are coupled together, two kinds of nanostructured particles were combined by mutual electrostatic adsorption. The PL of the QDs could then be used as the light source to excite the LSPR of the GNRs in a way that the QD PL is enhanced when the GNR LSPR interacts with the QD PL plane wave.

As shown in Figure 2a, from the FDTD simulation results we can see that (1) the highest PL enhancement, at $630 \mathrm{~nm}$, occurred when $d$ ( $d$ is the dipole-source center to GNR-center length) was set at $25 \mathrm{~nm}$ and (2) there is a slight enhancement at $526 \mathrm{~nm}$, which is the short axis of the GNRs. That means when

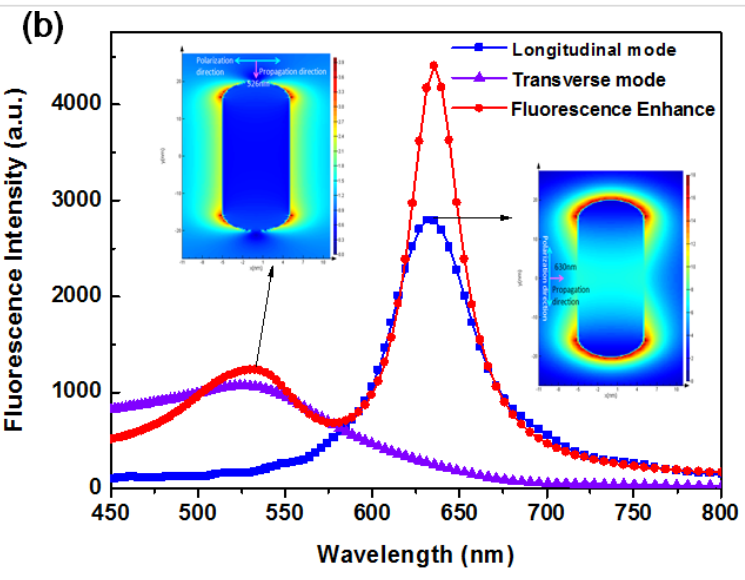

Figure 2: The simulation results of (a) photoluminescence enhancement as a function of wavelength and (b) wavelength and photoluminescence of GNRs under polarized light. The inset images inserted are the FDTD simulation of the electric field intensity distribution (indicated by the color bar) of the gold nanorods. 
the QD emission is overlaid with the longer absorption peak of the GNRs, the PL enhancement will be maximized. $5 \mathrm{~nm}$ was found to be the optimal distance between GNRs and QDs.

To more clearly determine that the dipole source is the actual source of the PL enhancement, we use light polarization directed along the long and short axes of the GNRs to stimulate the longitudinal and transverse modes, respectively. As shown in Figure 2b, which shows the FDTD simulation results, we can see that the peak at $630 \mathrm{~nm}$ (corresponding to PL enhancement) comes from GNR longitudinal excitation. The peak at $526 \mathrm{~nm}$ originates from the transverse mode GNR excitation. This enhanced photoluminescence is due to the PL emission and LSPR of the QDs and as a far-field dipole.

The size and morphology of the GNRs and GNR@CdSe/ZnS was characterized using transmission electron microscopy (TEM), and the results are shown in Figure $3 \mathrm{a}$ and $3 \mathrm{~b}$. The average diameter of the $\mathrm{CdSe} / \mathrm{ZnS}$ QDs is $8 \pm 1 \mathrm{~nm}$, where the corresponding TEM image is shown in Supporting Information
File 1, Figure S2. The aspect ratio (length/diameter) is approximately 2.2, where the short and long axes length of the nanorods is around $17 \mathrm{~nm}$ and $38 \mathrm{~nm}$, respectively, and the average distance between GNRs and QDs was around $5 \mathrm{~nm}$, as shown in Figure 3b. The energy dispersive X-ray spectroscopy (EDS) pattern of GNR@CdSe/ZnS is given in Figure 3c. The result was consistent with the atomic ratio of the structure of GNR@CdSe/ZnS. The presence of Cd, Au, Zn, and Se is clear, and in our work, $\mathrm{CdCl}_{2}$, Se powder, $\mathrm{ZnCl}_{2}$, and $\mathrm{Na}_{2} \mathrm{~S}$ were used as $\mathrm{Cd}, \mathrm{Se}, \mathrm{Zn}$, and $\mathrm{S}$ sources, respectively. On the other hand, the Au peak comes from the GNRs. The results agree well with the synthesis chemical ratio, as explained in the Experimental section, suggesting that GNR@CdSe/ZnS nanoparticles were successfully synthesized by the described synthetic route.

The absorption and PL spectrum of CdSe/ZnS QDs and GNRs and the PL spectra of CdSe/ZnS QDs and GNR@CdSe/ZnS were acquired at room temperature as they were prepared, and the spectrum of water was measured as a reference. Figure $4 \mathrm{a}$ shows the absorption spectrum of $\mathrm{CdSe} / \mathrm{ZnS}$ GNRs and

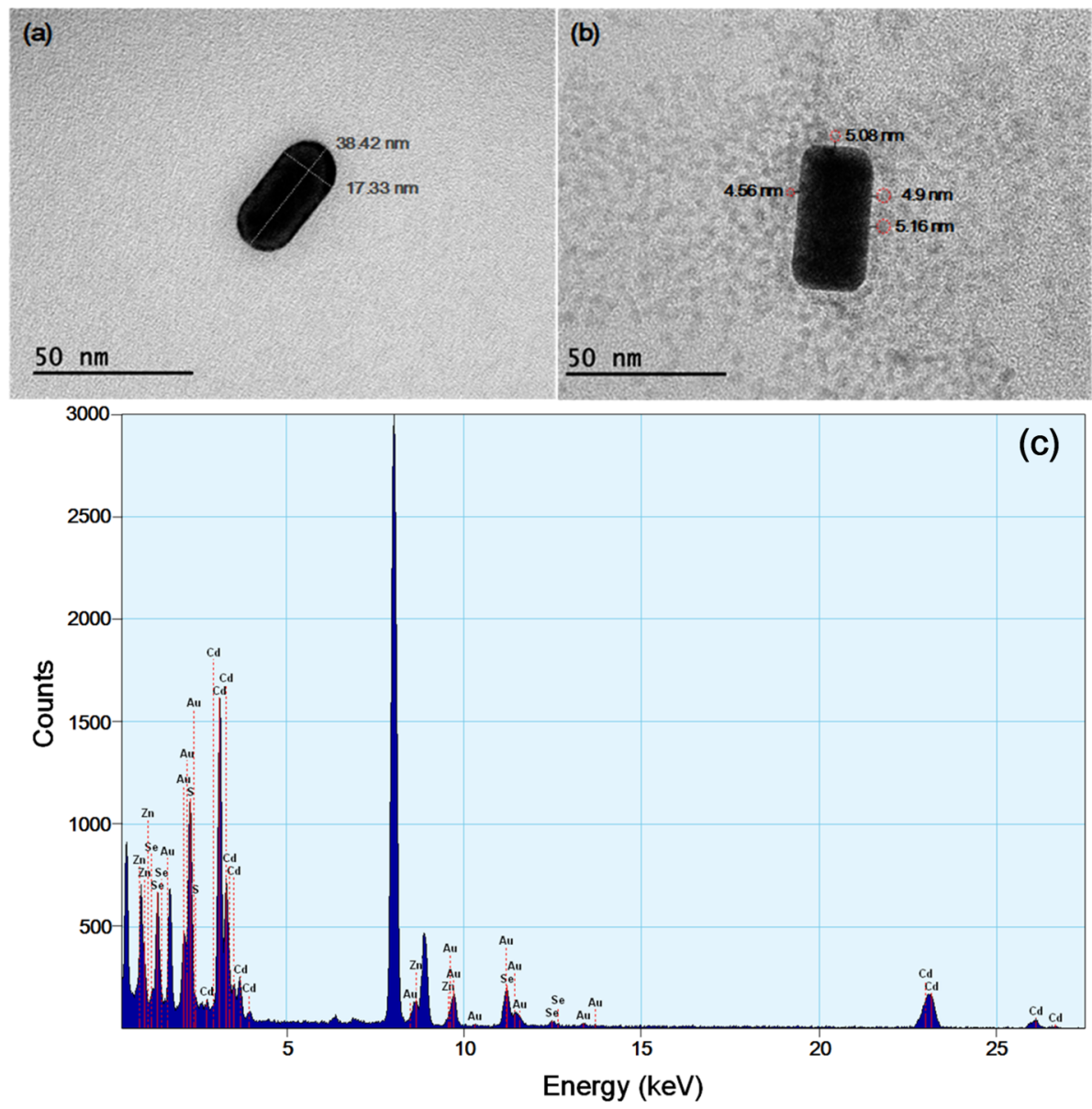

Figure 3: (a) TEM image of a GNR; (b) TEM image of GNR@CdSe/ZnS; (c) energy dispersive X-ray spectroscopy pattern of GNR@CdSe/ZnS. 

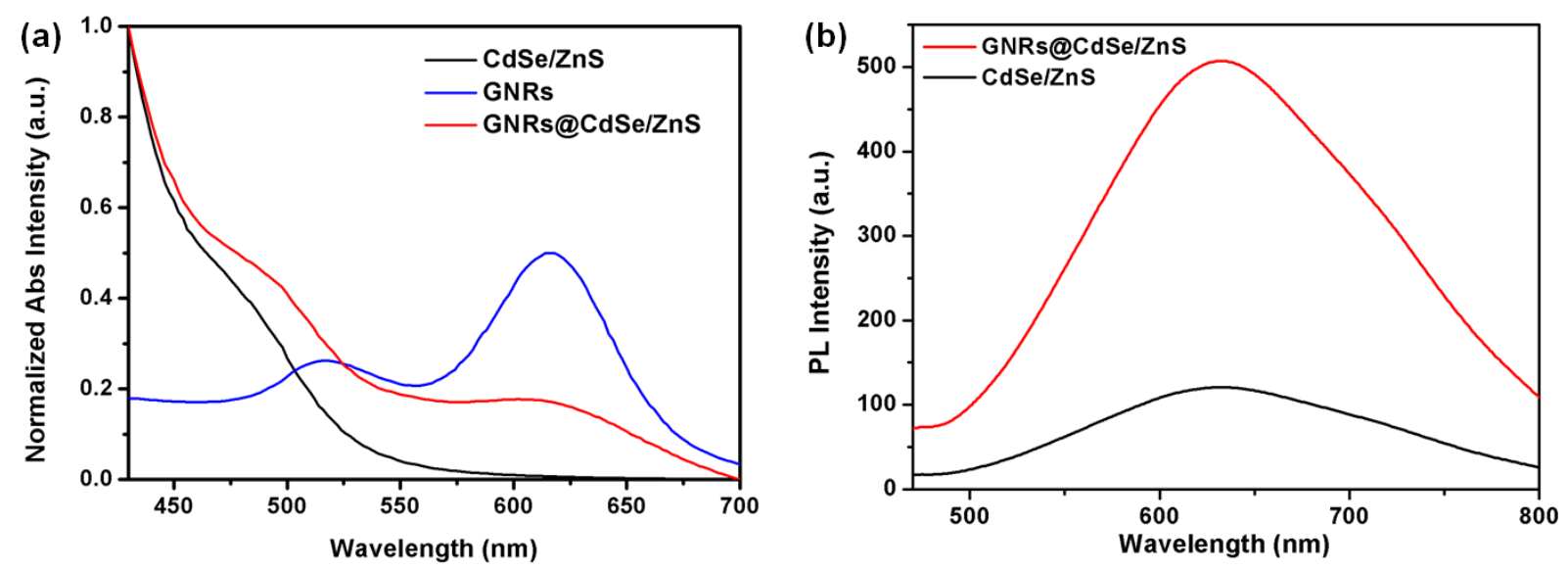

Figure 4: (a) Absorption spectrum of CdSe/ZnS, GNRs, and GNR@CdSe/ZnS; (b) PL spectrum of CdSe/ZnS and GNR@CdSe/ZnS.

GNR@CdSe/ZnS, where it can be seen that there are two peaks in the GNR absorption spectrum: the transverse surface plasmon resonance (TSPR) peak located at $510 \mathrm{~nm}$ and the LSPR peak located at $628 \mathrm{~nm}$. Figure $4 \mathrm{~b}$ shows the PL spectrum of $\mathrm{CdSe} / \mathrm{ZnS}$ QDs where the peak emission is around $630 \mathrm{~nm}$ and the GNR absorption overlaps with the emission of the CdSe/ZnS QDs. The PL emission intensity of GNR@CdSe/ $\mathrm{ZnS}$ was more than four times that of the CdSe/ZnS QDs, which implies that after binding with GNRs the PL intensity of CdSe/ ZnS QDs was enhanced.

We performed additional optical characterization of a single GNR@CdSe/ZnS nanoparticle at room temperature. We diluted the GNR@CdSe/ZnS solution then placed it on glass to obtain a single particle per $1 \times 1 \mu \mathrm{m}$ area. The experimental microspectroscopy equipment was used to collect the integrated, white light, dark-field scattering, as well as the PL spectra and the atomic force microscopy techniques. We measured the scattering and the PL emission signal from a single particle. The measurements were taken under continuous wave laser excitation at a wavelength of $532 \mathrm{~nm}$ with a laser power of about $100 \mu \mathrm{W}$. The confocal scanning PL image of GNR@CdSe/ZnS is plotted in Figure 5, which was acquired from a sample region of $40 \times 40 \mu \mathrm{m}$, where each dark spot corresponds to a single GNR@CdSe/ZnS nanoparticle.

Figure 5a shows the experimental PL and scattering spectrum of single particles of CdSe/ZnS QDs and GNR@CdSe/ZnS. Figure 5b shows optical confocal scanning images of GNR@CdSe/ZnS. When the GNRs were added to the QD solution, the PL emission intensity of GNR@CdSe/ZnS increased two times as compared with $\mathrm{CdSe} / \mathrm{ZnS}$. Additionally, compared to the PL spectrum in Figure 4b, the PL enhancement of a single particle is lower than that of the sample in the ensemble
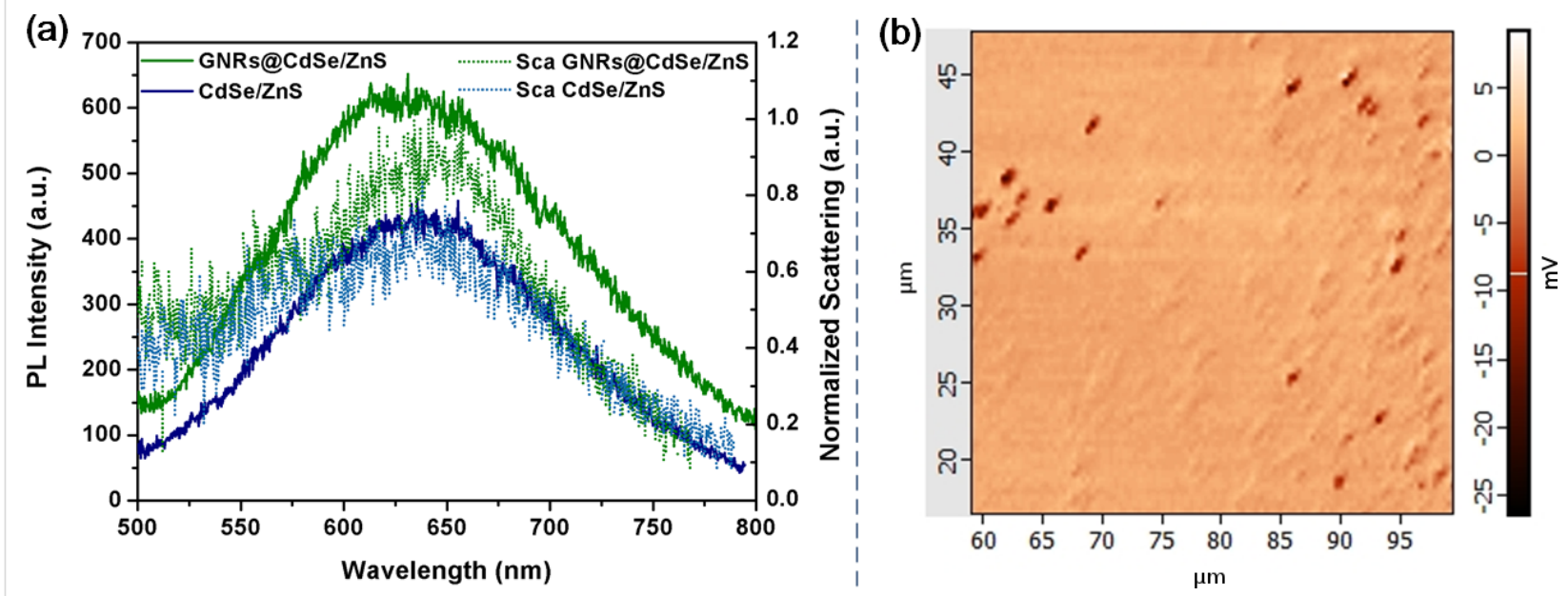

Figure 5: (a) PL and scattering spectrum from single CdSe/ZnS and GNR@CdSe/ZnS nanoparticles; (b) optical confocal scanning images of GNR@ $\mathrm{CdSe} / \mathrm{ZnS}$. 
solution. Although still bright, this test also confirmed that a single GNR@CdSe/ZnS nanoparticle could serve as a single photon source.

PL lifetime is also an important parameter in studying the photoelectric properties and surface chemistry of nanoparticles. A multiexponential decay curve provides evidence of the presence of nonradioactive or radiative recombination. The lifetime of GNR@CdSe/ZnS and CdSe/ZnS was obtained by using a fluorescence lifetime spectrometer (FLS980) with a detection wavelength range of 200 to $1700 \mathrm{~nm}$ and an excitation wavelength of $450 \mathrm{~nm}$. The spectra are shown in Figure 6.

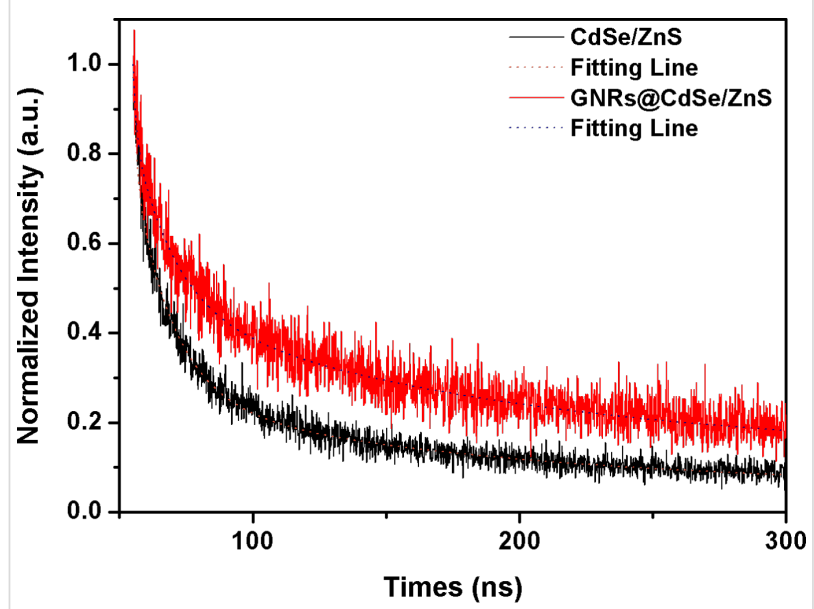

Figure 6: Photoluminescence lifetime spectrum of $\mathrm{CdSe} / \mathrm{ZnS}$ and GNR@CdSe/ZnS.

These lifetime measurements confirmed that the lifetime of GNR@CdSe/ZnS was longer than that of CdSe/ZnS, as shown by the data in Table 1 . Here, $B$ is a percentage of the lifetime and $t$ is the lifetime. From Figure 4 we can see that the band edge of the QDs is near $500 \mathrm{~nm}$ and the band edge of our CdSe/ $\mathrm{ZnS}$ sample is calculated to be about $2.48 \mathrm{eV}$, which is larger than the value of $1.76 \mathrm{eV}$ for $\mathrm{CdSe}$, thus the $\mathrm{PL}$ of $\mathrm{CdSe} / \mathrm{ZnS}$ is surface defect emission. This greater lifetime resulted from the trapping states caused by surface defects located within the bandgap, which led to a rise of nonradioactive recombination. When the GNRs were doped with CdSe/ZnS QDs, more radiative and nonradioactive processes occurred at the particle surface, which contributed to an enhanced lifetime.
To make this multifunctional nanomaterial more biocompatible and specific to the cancer cell, we conjugated the FA with GNR@CdSe/ZnS nanoparticles. Firstly, we performed a colloidal stability study in which their hydrodynamic sizes were monitored using the dynamic light scattering technique at room temperature. The results shown in Figure 7 indicated relatively stable conditions of GNR@CdSe/ZnS@FA formulations when the $\mathrm{pH}$ values were 4,7 , and 9 , and that the samples were relatively stable in the alkaline environment. The size of GNR@CdSe/ZnS@FA did not show significant change during the measurement, but when the $\mathrm{pH}$ value changed to 12, the size of GNR@CdSe/ZnS@FA increased. The size increase was caused by aggregation, which was due to the high $\mathrm{pH}$ solution being rich in hydroxide ions $\left(\mathrm{OH}^{-}\right)$, which facilitates the carboxyl groups of the surface of nanoparticles. However, still below $300 \mathrm{~nm}$, this size is also suitable for bioimaging, and the results suggest that the GNR@CdSe/ZnS@FA sample could be applied in biological applications.

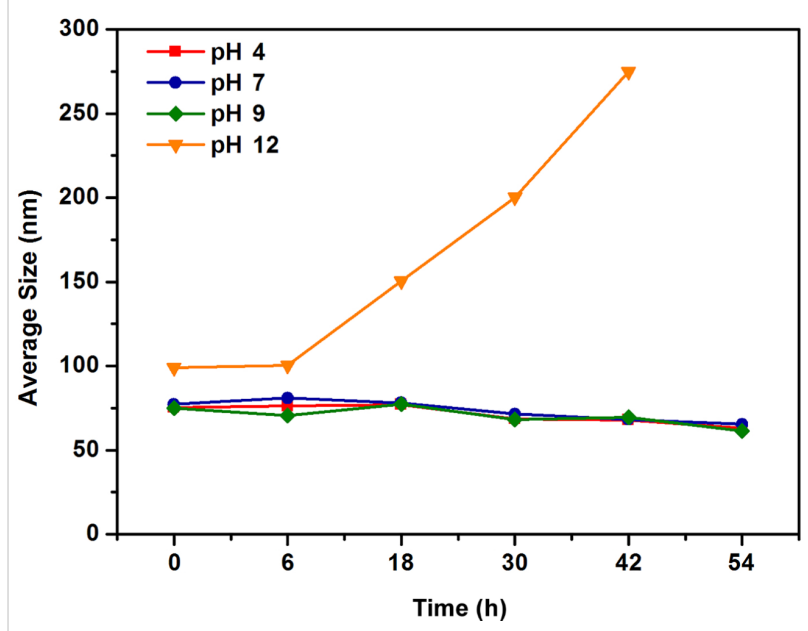

Figure 7: The colloidal stability of GNR@CdSe/ZnS@FA.

Over the past few years, the synthesis of CdSe and CdSe/ZnS QDs for in vitro imaging has received significant attention. To confirm that GNR@CdSe/ZnS can be used in cell imaging studies, after conjugation with FA, we performed a MCF-7 breast cancer cell targeting imaging study, and the results as shown in Figure 8. Robust cellular uptake could be obtained from the CdSe/ZnS@FA and GNR@CdSe/ZnS@FA treated samples. The obvious luminescence and staining appearing in

Table 1: Lifetime data of the samples.
\begin{tabular}{llllllll} 
Sample & $B_{1}$ & $t_{1}(\mathrm{~ns})$ & $B_{2}$ & $t_{2}$ (ns) & $B_{3}$ & $t_{3}$ (ns) & Average lifetime (ns) \\
\hline CdSe/ZnS & 0.0702 & 1.1411 & 0.2403 & 14.885 & 0.6896 & 128.5203 & 92.2753 \\
GNR@CdSe/ZnS & 0.0342 & 1.1047 & 0.1303 & 15.5347 & 0.8354 & 134.5797 & 114.5012
\end{tabular}




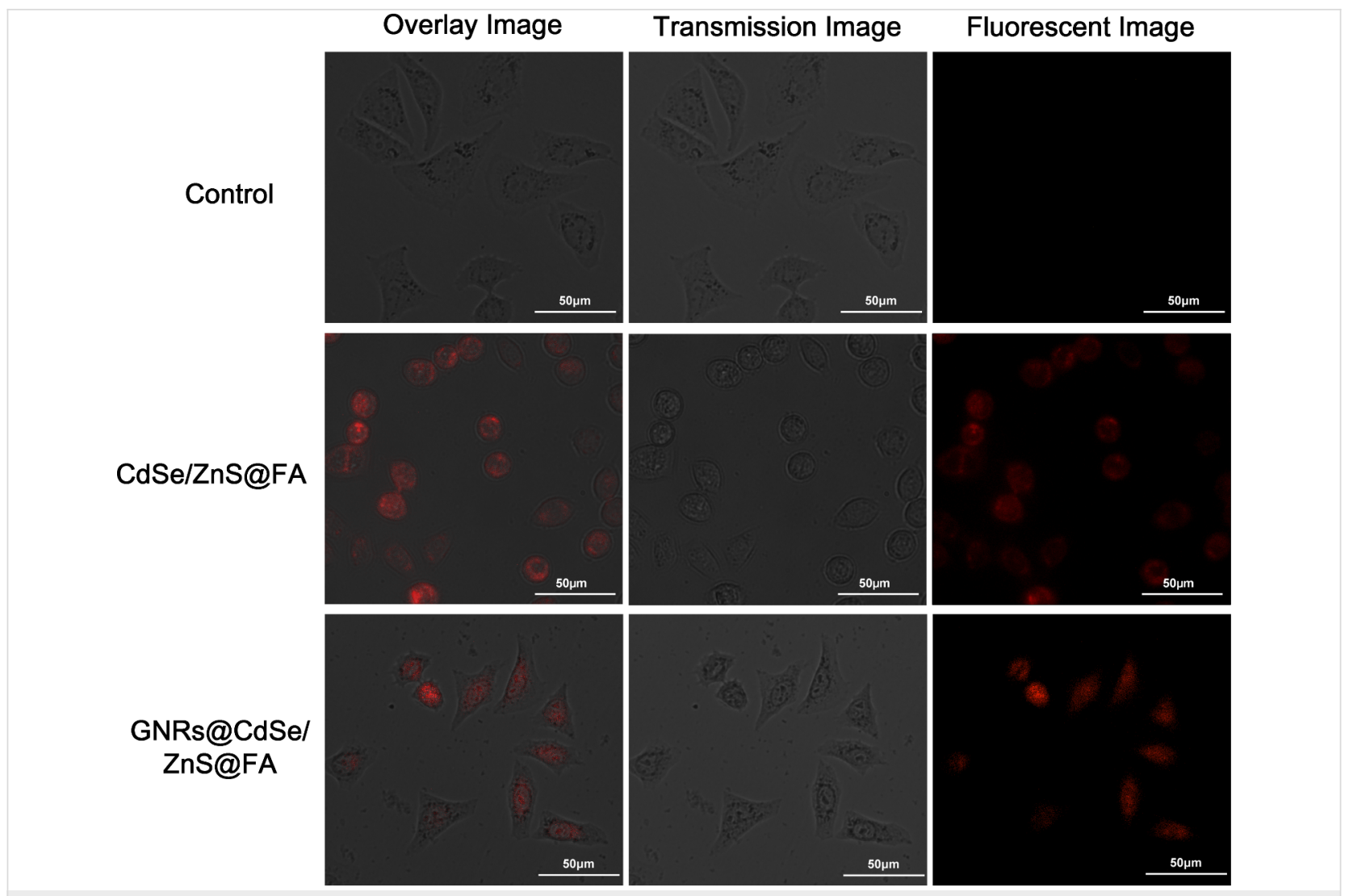

Figure 8: Microscopy images of MCF-7 breast cancer cell labelled with CdSe/ZnS@FA and GNR@CdSe/ZnS@FA.

Figure 8 was due to the accumulation of functionalized nanoparticles in the cells, and there was no sign of any damage to the cell, demonstrating passive uptake in MCF-7 breast cancer cells using CdSe/ZnS@FA and GNR@CdSe/ZnS@FA. However, because the PL intensity and cell morphology of GNR@CdSe/ $\mathrm{ZnS} @ \mathrm{FA}$ is better than $\mathrm{CdSe} / \mathrm{ZnS} @ \mathrm{FA}$, the in vitro imaging results confirm that these nanoparticles are suitable to be used for near-infrared imaging and cancer therapy.

\section{Conclusion}

We have developed a multifunctional nanomaterial GNR@CdSe/ZnS and enhanced the PL intensity using the surface plasmon resonance of GNRs. The experimental results correlated well with the theoretical simulation. The results of the biological detection study indicated that this nanomaterial is biocompatible and that there is significant PL signal for cell imaging. This research is promising for future nanophotonics and biophotonics applications.

\section{Experimental}

\section{Materials and instrumentation}

Hexadecyltrimethylammonium bromide (CTAB, >98.0\%), L-ascorbic acid (BioUltra, $\geq 99.5 \%$ ), silver nitrate $\left(\mathrm{AgNO}_{3}\right.$, $>99 \%$ ), gold(III) chloride trihydrate $\left(\mathrm{HAuCl}_{4} \cdot 3 \mathrm{H}_{2} \mathrm{O}, 99 \%\right)$, 3-mercaptopropionic acid (MPA, $\geq 99 \%), N$-ethyl- $N$ '-(3dimethylaminopropyl)carbodiimide (EDC, 99\%), and $\mathrm{N}$-hydroxysuccinimide (NHs, 99\%), cadmium chlorideanhydrous $\left(\mathrm{CdCl}_{2}, 99.999 \%\right)$, selenium powder ( $\left.\mathrm{Se}, 99.8 \%\right)$, sodium borohydride $\left(\mathrm{NaBH}_{4}, 99.99 \%\right)$, and zinc chloride anhydrous were purchased from Sigma-Aldrich. Sodium sulphide $\left(\mathrm{Na}_{2} \mathrm{~S}, 60-63 \%\right)$ was purchased from Acros Organics, and anhydrous ethanol, sodium hydroxide $(\mathrm{NaOH}, \mathrm{AR})$ and hydrochloric acid ( $\mathrm{HCl}, \mathrm{AR}$ ) were purchased from Sinopharm Chemical Reagent Co., Ltd. All chemicals were used as received without further purification. Deionized (DI) water used in all the studies was purified by a Milli-Q water purification system.

\section{Characterization}

The PL emission spectrum of the QDs was measured with a Cary Eclipse fluorescence spectrophotometer (Agilent, CA, USA) at an excitation wavelength of $430 \mathrm{~nm}$. UV-visible absorption spectra were collected using a Cary 5000 spectrophotometer (Agilent, CA, USA). The lifetime of the nanoparticles was measured with an FLS980 spectrometer (Edinburgh instruments, UK). The morphology and size of the GNRs and QDs were obtained with an FEI Tecnai G2 F20 S-TWIN TEM operating at an accelerating voltage of $200 \mathrm{kV}$, and the samples were loaded into a quartz cell for the measurements. The TEM 
specimens were prepared by drop casting the sample dispersion onto an amorphous carbon-coated 300 mesh copper grid.

\section{Synthesis of GNRs}

To synthesize the GNRs, the seed-mediated growth method in CTAB solution was applied, as previously discussed [30,31] The seed solution was prepared using $5 \mathrm{~mL}$ of a $0.2 \mathrm{M} \mathrm{CTAB}$ solution and $5 \mathrm{~mL}$ of $0.1 \mathrm{mM} \mathrm{HAuCl}_{4}$. Following this, $0.6 \mathrm{~mL}$ of ice-cold $0.01 \mathrm{M} \mathrm{NaBH}_{4}$ solution was quickly added to the $\mathrm{HAuCl}_{4}{ }^{-} \mathrm{CTAB}$ solution and vigorously stirred for 3-5 min. This caused the solution color to change from yellow to light brown. The solution was stored at $37^{\circ} \mathrm{C}$ for $30 \mathrm{~min}$ before use. The growth solution was prepared by mixing $800 \mu \mathrm{L}$ of $25 \mathrm{mM}$ $\mathrm{HAuCl}_{4}$ and $20 \mathrm{~mL}$ of HPLC water. Following this, $400 \mu \mathrm{L}$ of $4.0 \mathrm{mM} \mathrm{AgNO}_{3}$ solution was added to the $\mathrm{HAuCl}_{4}$ solution, and then $20 \mathrm{~mL}$ of $0.2 \mathrm{M} \mathrm{CTAB}$ and $800 \mu \mathrm{L}$ of $0.08 \mathrm{M}$ ascorbic acid were added. This solution was kept in a water bath at $37^{\circ} \mathrm{C}$, and the solution became clear and colorless after gentle stirring. $96 \mu \mathrm{L}$ of the seed solution was added to the growth solution, and it was then left undisturbed at $37{ }^{\circ} \mathrm{C}$ for $24 \mathrm{~h}$. The final solution was centrifuged at $8000 \mathrm{rpm}$ for $10 \mathrm{~min}$ twice then redispersed in $0.02 \mathrm{M} \mathrm{CTAB}$ solution for later use.

\section{Synthesis of CdSe/ZnS QDs}

A selenium precursor was prepared by reducing $19.4 \mathrm{mg}$ of selenium powder with $40 \mathrm{mg}$ of sodium borohydride $\left(\mathrm{NaBH}_{4}\right)$ in $1 \mathrm{~mL}$ of nitrogen-saturated DI water at room temperature. The mixture was stirred for $1-2 \mathrm{~h}$ until it became colorless.

MPA-CdSe QDs were synthesized by the previously reported method. Briefly, $366 \mathrm{mg}$ of $\mathrm{CdCl}_{2}, 440 \mu \mathrm{L}$ of MPA, and $50 \mathrm{~mL}$ of nitrogen-saturated water were loaded into a three-necked flask under stirring. The $\mathrm{pH}$ was adjusted to 10 by dropwise addition of sodium hydroxide solution. The Se precursor was then injected into the mixture under nitrogen atmosphere, and the reaction mixture was slowly heated under nitrogen atmosphere to $98^{\circ} \mathrm{C}$. After $2 \mathrm{~h}$, the mixture solution was collected as MPA$\mathrm{CdSe} \mathrm{QDs}$ for $\mathrm{ZnS}$ shell-coating synthesis. The $\mathrm{ZnCl}_{2}$ was prepared by loading $68 \mathrm{mg}$ of $\mathrm{ZnCl}_{2}, 100 \mu \mathrm{L}$ of MPA, and $30 \mathrm{~mL}$ of nitrogen-saturated water into a three-necked flask under stir- ring. The $\mathrm{pH}$ was adjusted to 10 by adding $1 \mathrm{M}$ of sodium hydroxide solution. The $\mathrm{ZnCl}_{2}$ solution was then added to the CdSe solution at $60{ }^{\circ} \mathrm{C}$, and after the mixture was heated to $90{ }^{\circ} \mathrm{C}, 60 \mathrm{mg}(2 \mathrm{~mL})$ of $\mathrm{Na}_{2} \mathrm{~S}$ was added to the $\mathrm{ZnCl}_{2}$-CdSe solution and maintained at $98{ }^{\circ} \mathrm{C}$ for $1 \mathrm{~h}$. Finally, the solution was cooled to room temperature and separated by the addition of ethanol and two cycles of centrifugation.

\section{Synthesis of GNR@CdSe/ZnS nanoparticles}

The GNR solution ( $5 \mathrm{~mL}, 5 \mathrm{OD} / \mathrm{mL}$ ) was centrifuged at $8000 \mathrm{rpm}$ for $10 \mathrm{~min}$ and the supernatant was discarded. The GNR precipitate was redispersed in HPLC water to obtain $0.1 \mathrm{OD} / \mathrm{mL}$. Subsequently, $1 \mathrm{~mL}$ of the CdSe/ZnS ( $1 \mathrm{OD} / \mathrm{mL}$ ) mixture was added to $10 \mathrm{~mL}$ of the GNR solution, and the mixture was left under stirring overnight to obtain the GNR@CdSe/ $\mathrm{ZnS}$ composite nanoparticles.

\section{Synthesis of GNR@CdSe/ZnS@FA nanoparticles}

From the stock solution, $2 \mathrm{~mL}$ of $4 \mathrm{mg} / \mathrm{mL}$ aqueous GNR@CdSe/ZnS dispersion was mixed with $400 \mu \mathrm{L}$ of $10 \mathrm{mM}$ EDC solution and incubated for $2 \mathrm{~min} .400 \mu \mathrm{L}$ of $10 \mathrm{mM} \mathrm{NHs}$ was then added to the mixture and stirred for $10 \mathrm{~min}$. Next, $1.2 \mathrm{~mL}$ of folic acid in DMSO solution $(2 \mathrm{mg} / \mathrm{mL})$ was added to this mixture and stirred at room temperature for $2 \mathrm{~h}$ to allow the folic acid to covalently couple with the nanoparticles. After two hours of stirring, the bioconjugated nanoparticles were purified (removing excess by-products) via centrifugation. The QD precipitate was redispersed in HPLC water for the bioimaging studies.

\section{Simulation study of photoluminescence enhancement}

The simulation study was based on Parseval's equation, where the electromagnetic fields can be expressed as described in Equation 1 and Equation 2, and the dipole integral is according to the perpendicular [26].

The normalized total output power of photoluminescence of can be expressed as [26]

$$
\begin{gathered}
H=-\frac{k_{0}^{3} c_{0} \vec{p}}{4 \pi} \hat{\varphi}_{\mathrm{r}} \int_{0}^{\infty} \mathrm{d} n_{\mathrm{p}} \frac{n_{\rho}^{2}}{\sqrt{n_{\mathrm{d}}^{2}-n_{\rho}^{2}}} J_{1}\left(k_{0} n_{\rho} \rho\right)\left(a e^{i k_{0} n_{z} z}+b e^{i k_{0} n_{z} z}\right) \\
E=-\frac{i u_{0} c_{0}^{2} k_{0}^{3} \vec{p}}{4 \pi n n_{\mathrm{d}}} \int_{0}^{\infty} \mathrm{d} n_{\rho} \frac{n_{\rho}}{\sqrt{n_{\mathrm{d}}^{2}-n_{\rho}^{2}}}\left[\begin{array}{l}
\left.i \hat{\rho}_{\mathrm{r}} n_{z} J_{1}\left(k_{0} n_{\rho} \rho\right)\left(a e^{i k_{0} n_{z} z}-b e^{i k_{0} n_{z} z}\right)\right] \\
\left.-\hat{z} n_{\rho} J_{0}\left(k_{0} n_{\rho} \rho\right)\left(a e^{i k_{0} n_{z} z}+b e^{i k_{0} n_{z} z}\right)\right]
\end{array}\right.
\end{gathered}
$$




$$
\begin{gathered}
p=\frac{3 n^{5} \sin (\theta)^{2} \cos (\theta)^{2}}{8 \pi n_{\mathrm{d}}^{3}\left|n_{\mathrm{d}}^{2}-n^{2} \sin (\theta)^{2}\right|}||^{2} \\
\frac{p_{z}}{p_{1}}=\frac{3}{4} \mathfrak{R}\left[\frac{n^{*}}{n_{1}^{3} n} \int_{0}^{\infty} \mathrm{d} n_{\rho} \frac{n_{\rho}^{3} \sqrt{n^{2}-n_{\rho}^{2}}}{\left|n_{1}^{2}-n_{\rho}^{2}\right|}(a-b)\left(a^{*}+b^{*}\right)\right] \\
\frac{p_{x y}}{p_{1}}=\frac{3}{8} \Re\left[\int_{0}^{\infty} \mathrm{d} n_{\rho} n_{\rho}\left(\frac{n^{*}}{n_{1}^{3} n}+\frac{1}{n_{1}\left|n_{1}^{2}-n_{\rho}^{2}\right|}\right) \sqrt{n^{2}-n_{a}^{2}}(a-b)\left(a^{*}+b^{*}\right)\right]
\end{gathered}
$$

We also simulated the PL enhancement of GNR@CdSe/ZnS using FDTD software, and we used these simulation results to further study the PL enhancement caused by LSPR coupling effect. The FDTD simulation results show that the quantum mechanical decay rate of the inhomogeneous environment is related to the classical power radiated by the dipole in the same environment, which only occurs in the radiation of an atomic dipole transition. Specifically, we have the following relationship:

$$
\frac{\Gamma_{\mathrm{rad}}}{\Gamma_{\mathrm{rad}}^{0}}=\frac{P}{P_{0}}
$$

where $\Gamma_{\text {rad }}$ is the decay rate, $P$ is the radiated power in an inhomogeneous environment (with the nanoparticle near the dipole source), $\Gamma_{\text {rad }}^{0}$ is the decay rate, and $P_{0}$ is the power radiated in a homogeneous environment (only the dipole source). When the isolated emitter is coupled to the nanoantenna, its quantum efficiency is modified as,

$$
\eta_{(\omega)}=\eta_{0(\omega)} /\left[\left(1-\eta_{0(\omega)}\right) / F_{(\omega)}+\eta_{0(\omega)} / \eta_{a(\omega)}\right]
$$

In this relation,

$$
\text { Purcell factor: } F_{(\omega)}=\Gamma_{\mathrm{rad}} / \Gamma_{\mathrm{rad}}^{0}
$$

$$
\text { Antenna efficiency: } \eta_{a(\omega)}=\Gamma_{\mathrm{rad}} /\left(\Gamma_{\mathrm{rad}}+\Gamma_{\mathrm{nrad}}\right)
$$

The PL enhancement, $S / S_{0}$, is then

$$
\frac{S}{S_{0}}=\frac{\eta_{(\omega)}|p E|^{2}}{\eta_{0(\omega)}\left|p E_{0}\right|^{2}} \approx F_{(\omega)} \frac{\eta_{(\omega)}}{\eta_{0(\omega)}}
$$

where $p$ is the transition electric dipole moment. From the above equations, we can see the PL enhancement effected by the $F_{(\omega)}$ and $\eta_{a(\omega)}$. Here $\eta_{0(\omega)}$ is the quantum efficiency related to the enhancement as a reference [22,23].

For FDTD simulations we use a dipole source to simulate the QDs, which emit in the wavelength range from $450 \mathrm{~nm}$ to $800 \mathrm{~nm}$ with polarization along the long axis direction $z$. $d$ is the distance between the dipole source center and the GNR center, and the length of the GNRs was fixed at $40 \mathrm{~nm}$.

\section{In vitro cell imaging study}

MCF-7 breast cancer cells (American Type Culture Collection) were cultured with Dulbecco's Modified Eagle's Medium (DMEM, Hyclone), supplemented with $10 \%$ fetal bovine serum (FBS, Hyclone), penicillin (100 $\mu \mathrm{g} \mathrm{mL}^{-1}$, Gibco), and streptomycin $\left(100 \mu \mathrm{g} \mathrm{mL}^{-1}\right.$, Gibco) in a humidified environment $\left(37{ }^{\circ} \mathrm{C}, 5 \% \mathrm{CO}_{2}\right)$. Before treating with nanoparticles, the cells were seeded onto cover glass in a six-well plate with DMEM. The prepared CdSe/ZnS@FA QDs and GNR@CdSe/ZnS@FA nanoparticles were then diluted with PBS buffer (pH 7.2) solution to a concentration of $500 \mu \mathrm{g} / \mathrm{mL}$. Next, the cells were treated with the CdSe/ZnS@FA QDs and GNR@CdSe/ ZnS@FA nanoparticles for 4 h. After 4 h of incubation, the treated cells were washed with PBS buffer three times. A Leica DMI 3000 inverted microscope with a $10 \times$ lens was used for the cell imaging study, and the excitation and emission wavelengths were $532 \mathrm{~nm}$ and $630 \mathrm{~nm}$, respectively.

\section{Supporting Information}

\section{Supporting Information File 1}

Relative cell viability of MCF-7 breast cancer cells treated with different concentrations $(6.25-100 \mu \mathrm{g} / \mathrm{mL})$ of GNR@CdSe/ZnS for 24 h. TEM image of CdSe/ZnS QDs. [https://www.beilstein-journals.org/bjnano/content/ supplementary/2190-4286-10-3-S1.pdf]

\section{Acknowledgements}

This work was supported by the National Natural Science Foundation of China (Grant No. 61701493, 61722508, 61525503, 61378091, 81727804), the Postdoctoral Research Funding Program of Jiangsu Province (2018K004B), the Advance Research Project of Weapon and Equipment (6140414020102), the Developing Project of Science and Technology of Jilin Province (20180519017JH). Science Foundation for Young Scientists of Changchun University of Science and Technology (XQNJJ-2016-10). Parts of this work were supported by the International Joint Research Center for Nanophotonics and Biophotonics, School of Science, Changchun University of 
Science and Technology, and Nanophotonics and Biophotonics Key Laboratory of Jilin Province, P.R. China (20140622009J).

\section{ORCID ${ }^{\circledR}$ iDs}

Siyi Hu - https://orcid.org/0000-0002-0686-5182 Junle Qu - https://orcid.org/0000-0001-7833-4711

\section{References}

1. Razgoniaeva, N.; Lambright, S.; Sharma, N.; Acharya, A.; Khon, E.; Moroz, P.; Razgoniaev, A.; Ostrowski, A.; Zamkov, M.

J. Phys. Chem. C 2015, 119, 15562-15571. doi:10.1021/acs.jpcc.5b04608

2. Prasad, P. Nanophotonics; John Wiley \& Sons, Inc.: New York, NY, U.S.A., 2004; p 208. doi:10.1002/0471670251

3. Hu, S.; Zeng, S.; Zhang, B.; Yang, C.; Song, P.; Hang Danny, T. J.; Lin, G.; Wang, Y.; Anderson, T.; Coquet, P.; Liu, L.; Zhang, X.; Yong, K.-T. Analyst 2014, 139, 4681-4690. doi:10.1039/c4an00773e

4. Liu, L.-w.; Hu, S.-y.; Pan, Y.; Zhang, J.-q.; Feng, Y.-s.; Zhang, X.-h. Beilstein J. Nanotechnol. 2014, 5, 919-926. doi:10.3762/bjnano.5.105

5. Kim, K. S.; Hur, W.; Park, S.-J.; Hong, S. W.; Choi, J. E.; Goh, E. J.; Yoon, S. K.; Hahn, S. K. ACS Nano 2010, 4, 3005-3014. doi:10.1021/nn100589y

6. Pons, T.; Medintz, I. L.; Sapsford, K. E.; Higashiya, S.; Grimes, A. F.; English, D. S.; Mattoussi, H. Nano Lett. 2007, 7, 3157-3164. doi:10.1021/nl071729

7. Shevchenko, E. V.; Ringler, M.; Schwemer, A.; Talapin, D. V.; Klar, T. A.; Rogach, A. L.; Feldmann, J.; Alivisatos, A. P. J. Am. Chem. Soc. 2008, 130, 3274-3275. doi:10.1021/ja710619s

8. Mohammadi, A.; Kaminski, F.; Sandoghdar, V.; Agio, M. J. Phys. Chem. C 2010, 114, 7372-7377. doi:10.1021/jp9094084

9. Mohammadi, A.; Sandoghdar, V.; Agio, M. New J. Phys. 2008, 10 , 105015. doi:10.1088/1367-2630/10/10/105015

10. Rogobete, L.; Kaminski, F.; Agio, M.; Sandoghdar, V. Opt. Lett. 2007, 32, 1623-1625. doi:10.1364/ol.32.001623

11. Vivas, M. G.; Cury, J. F.; Schiavon, M. A.; Mendonca, C. R. J. Phys. Chem. C 2013, 117, 8530-8535. doi:10.1021/jp400956k

12. Mokari, T.; Rothenberg, E.; Popov, I.; Costi, R.; Banin, U. Science 2004, 304, 1787-1790. doi:10.1126/science.1097830

13. Menagen, G.; Macdonald, J. E.; Shemesh, Y.; Popov, I.; Banin, U. J. Am. Chem. Soc. 2009, 131, 17406-17411. doi:10.1021/ja9077733

14. AbouZeid, K. M.; Mohamed, M. B.; El-Shall, M. S. Small 2011, 7, 3299-3307. doi:10.1002/smll.201100688

15. Rosen, S.; Schwartz, O.; Oron, D. Phys. Rev. Lett. 2010, 104, 157404. doi:10.1103/physrevlett.104.157404

16. Bothun, G. D.; Rabideau, A. E.; Stoner, M. A. J. Phys. Chem. B 2009, 113, 7725-7728. doi:10.1021/jp9017458

17. Gil, P. R.; Parak, W. J. ACS Nano 2008, 2, 2200-2205. doi:10.1021/nn800716j

18. Ehamparam, R.; Pavlopoulos, N. G.; Liao, M. W.; Hill, L. J.; Armstrong, N. R.; Pyun, J.; Saavedra, S. S. ACS Nano 2015, 9 , 8786-8800. doi:10.1021/acsnano.5b01720

19. Ji, W.; Jing, P.; Zhao, J.; Liu, X.; Wang, A.; Li, H. Nanoscale 2013, 5 , 3474-3480. doi:10.1039/c3nr34168b

20. Araci, Z. O.; Shallcross, C. R.; Armstrong, N. R.; Saavedra, S. S. J. Phys. Chem. Lett. 2010, 1, 1900-1905. doi:10.1021/jz100475j

21. Fang, Y.; Chang, W.-S.; Willingham, B.; Swanglap, P.; Dominguez-Medina, S.; Link, S. ACS Nano 2012, 6, 7177-7184. doi:10.1021/nn3022469
22. Lu, G.; Zhang, T.; Li, W.; Hou, L.; Liu, J.; Gong, Q. J. Phys. Chem. C 2011, 115, 15822-15828. doi:10.1021/jp203317d

23. Zhang, T.; Lu, G.; Li, W.; Liu, J.; Hou, L.; Perriat, P.; Martini, M.; Tillement, O.; Gong, Q. J. Phys. Chem. C 2012, 116, 8804-8812. doi:10.1021/jp2125944

24. Nepal, D.; Drummy, L. F.; Biswas, S.; Park, K.; Vaia, R. A. ACS Nano 2013, 7, 9064-9074. doi:10.1021/nn403671q

25. Saraf, D.; Kshirsagar, A. Phys. Chem. Chem. Phys. 2014, 16, 10823-10829. doi:10.1039/c4cp00069b

26. Chen, D.; Zhao, C.; Ye, J.; Li, Q.; Liu, X.; Su, M.; Jiang, H.; Amatore, C.; Selke, M.; Wang, X. ACS Appl. Mater. Interfaces 2015, 7, 18163-18169. doi:10.1021/acsami.5b05805

27. Dai, L.; Zhang, Q.; Li, J.; Shen, X.; Mu, C.; Cai, K. ACS Appl. Mater. Interfaces 2015, 7, 7357-7372. doi:10.1021/acsami.5b00746

28. Zhang, R.-Y.; Wang, Z.-Y.; Yang, X.-Q.; Xuan, Y.; Cheng, K.; Li, C.; Song, X.-L.; An, J.; Hou, X.-L.; Zhao, Y.-D. Nanotechnology 2018, 29 , 055101. doi:10.1088/1361-6528/aa9acc

29. Geszke, M.; Murias, M.; Balan, L.; Medjahdi, G.; Korczynski, J.; Moritz, M.; Lulek, J.; Schneider, R. Acta Biomater. 2011, 7, 1327-1338. doi:10.1016/j.actbio.2010.10.012

30. Law, W.-C.; Yong, K.-T.; Baev, A.; Prasad, P. N. ACS Nano 2011, 5, 4858-4864. doi:10.1021/nn2009485

31. Nikoobakht, B.; El-Sayed, M. A. Chem. Mater. 2003, 15, 1957-1962. doi:10.1021/cm020732।

\section{License and Terms}

This is an Open Access article under the terms of the Creative Commons Attribution License (http://creativecommons.org/licenses/by/4.0). Please note that the reuse, redistribution and reproduction in particular requires that the authors and source are credited.

The license is subject to the Beilstein Journal of Nanotechnology terms and conditions: (https://www.beilstein-journals.org/bjnano)

The definitive version of this article is the electronic one which can be found at: doi:10.3762/bjnano. 10.3 